\title{
Two-Temperature Generalized Thermopiezoelasticity for One Dimensional Problems - State Space Approach
}

\author{
H. M. Youssef ${ }^{1}$, E. Bassiouny ${ }^{2}$ \\ ${ }^{1}$ Faculty of Engineering, Umm Al-Qura University, PO. Box 5555, Makkah, Saudi Arabia \\ e-mail:yousefanne@yahoo.com \\ ${ }^{2}$ King Saud University, Faculty of Science, Department of Mathematics P.O.Box 83, 11942 Al-Kharj, Saudi Arabia \\ e-mail: essambassiouny@hotmail.com
}

(Received: 25 April 2007; revised: 8 February 2008; accepted: 21 February 2008; published online: 7 April 2008)

\begin{abstract}
The theory of two-temperature generalized thermoelasticity, based on the theory of Youssef is used to solve boundary value problems of one dimensional piezoelectric half-space with heating its boundary with different types of heating. The governing equations are solved in the Laplace transform domain by using state-space approach of the modern control theory. The general solution obtained is applied to a specific problems of a half-space subjected to three types of heating; the thermal shock type, the ramp type and the harmonic type. The inverse Laplace transforms are computed numerically using a method based on Fourier expansion techniques. The conductive temperature, the dynamical temperature, the stress and the strain distributions are shown graphically with some comparisons.
\end{abstract}

Key words: piezoelectric material, generalized thermoelasticity, state space, two-temperature

\section{Nomenclature:}

$A_{i j}-$ the components of relaxation time

$a$ - the two-temperature parameter

$C_{E}-$ specific heat at constant strain

$c_{i j k l}-\quad$ the elastic coefficients

$c_{0}-\sqrt{(\lambda+2 \mu) / \rho}-$ longitudinal wave speed

$D_{i}-$ the components of electric displacement

$d_{i}-$ the pyroelectric coefficient

$E_{i}-$ the components of electric field vector

$e_{i j}-$ the components of strain tensor

$h_{i j k}{ }^{-}$the piezoelectric coefficients

$k_{i j}$ - the components of thermal conductivity

$q_{i}-$ the components of the heat flux vector

$T$ - absolute temperature

$T_{0}-$ reference temperature

$t$ - time

$t_{0}-$ ramping time parameter

$u_{i}-$ components of displacement vector

$v_{i}-$ the electric potential function $\alpha-\gamma T_{0} /(\lambda+2 \mu)-$ dimensionless thermoelastic coupling constant

$\alpha_{T}-$ coefficient of linear thermal expansion

$\beta_{i k}$ - the components of dielectric tensor

$\gamma-(3 \lambda+2 \mu) \alpha_{T}$

$\Omega-$ the angular frequency of thermal vibration

$\delta_{i j}-$ Kronecker delta function

$\varepsilon-\gamma / \rho C_{E}-$ dimensionless mechanical coupling constant

$\zeta-$ the entropy

$\eta-\rho C_{E} / k-$ the thermal viscosity

$\theta \quad\left(T-T_{0}\right)-$ the dynamical temperature increment such that $\left|T-T_{0}\right| / T_{0} \ll 1$

$\lambda, \mu-$ Lamé's constants

$\rho-$ density

$\sigma_{i j}-$ components of stress tensor

$\sigma-$ the principal Stress component

$\tau_{0}-$ one relaxation time parameter

$\varphi-$ the conductive temperature

$\omega-$ dimensionless two-temperature parameter 


\section{INTRODUCTION}

For classical uncoupled and coupled theories of thermoelasticity, the heat conduction equations are of the diffusion types which lead to infinite speeds of propagation for heat waves contrary to physical observations. Widespread attention to eliminate this paradox has been given to thermoelasticity theories which admit a finite speed for the propagation of thermal waves. Many authors have formulated generalized theories involve a hyperbolic-type heat equation and are referred to as generalized thermoelasticity. Three generalizations to the coupled theory were introduced. The first theory was developed by Lord and Shulman [1]. In this theory they obtained a wave-type heat equation by modifying Fourier's law to contain the heat flux vector as well as its derivative and include one relaxation time. Since the heat equation of this theory is of the wave-type, it ensures finite speed of propagation for heat and elastic waves [2]. This eliminates the paradox accompanying the infinite speed of heat propagation in the classical theory and allows for the so called second sound effects in solids. Ignaczak contributes to the thermoelasticity with one relaxation by the proofs of uniqueness theorems under different conditions [3].

The second generalization to the coupled theory of elasticity is what is known as the theory of thermoelasticity with two relaxation times or the theory of temperature-rate dependent thermoelasticity. In this theory an entropy production inequality was proposed by Müller [4]. It represents restrictions on a class of constitutive equations. The Green and Lindsay (G-L) theory modifies both the energy equation and the Duhamel-Neuman relation and admits two relaxation times and modify all equations of the coupled theory, not only the heat equation. The classical Fourier's law of heat conduction is not violated if the medium under consideration has a center of symmetry $[5,6]$. They also obtained another version of this constitutive equations.

In the third theory [C-T] theory, Tzou [7] replace the Fourier law by an approximation to a modification of the Fourier law with two different translations for the heat flux and the temperature gradient. This theory is known as the dual-phase-lag thermoelasticity.

Piezoelectricity is the phenomenon whereby electric polarization is devoted in deformed materials. The effect of electro-mechanical coupling in such materials has immense potential in engineering applications. A good example is the use of this class of materials as sensors and actuators in micro- electro-mechanical system (MEMS), for instance, the piezoelectric accelerometer which triggers an airbag in ten of second during accident.
With the advent of new generation of electronic devices, their reliability and integrity are essential for safe operation. In addition, because these devices are to operate under various electro-thermo-mechanical conditions over a broad spectrum, their design and manufacturing represent a great challenge in engineering. In view of its versatility and important to engineering applications, we devoted our attention to piezoelectric ceramic which have been extensively used in many engineering applications. So, the theory of generalized thermo-piezoelectricity has been the object of numerous investigations in the last decades or so, concerning both its theoretical foundations and the applications.

The theory of generalized thermoelasticity was extended so as to involve electromagnetic media, piezoelectric in particular. The theory of thermo-piezoelectricity was first proposed by Mindlin [8]. He also derived governing equations of a thermo-piezoelectric plate [9]. Nowacki $[10,11]$ has studied the physical laws for the thermo-piezoelectric materials. Chandrasekharaiah [12] has generalized Mindlin's theory of thermo-piezoelectricity to account for the finite speed of propagation of thermal disturbances. Majhi [13] studied the transient thermal response of a semi-infinite piezoelectric rod subjected to a local heat source along the length direction, by introducing a potential function and applying the L-S theory. Sharma and Kumar [14] studied plane harmonic waves in piezo-thermoelastic materials. Bassiouny and Ghaleb have solved a one dimensional problem in the generalized theory of thermopiezoelasticity [15]. Tianhu et al. [16,17] discussed various thermal shock problems of piezoelectric plate by applying the L-S and G-L theories. Baljeet [18] used Green-Lindsay and Lord-Shulman theories for generalized thermo-piezoelectric solid to study the plane waves in a two-dimensional model.

Recently, Youssef [19] has improved the previous theories of the generalized thermoelasticity (G-L) and (L-S) with a new theory which depends on two distinct temperatures, that is, the conductive temperature and the thermodynamic temperature, this theory is called; Theory of twotemperature generalized thermoelasticity (Y-TTGTE). The uniqueness solution of the last theory has been derived also by Youssef.

The present work, use the state space approach and the two-temperature theory (Y-TTGTE) to study the effect of the presence of the heat conduction $\varphi$ in Fourier's law instead of the usual thermodynamic temperature on the behavior of the solutions in generalized thermo-piezoelectricity. Considering the (Y-TTGTE) model, the governing differential equations for two-temperature generalized thermo-piezoelectric solid are formulated. Laplace trans- 
form technique is applied to thermal shock of semi infinite piezoelectric rod to obtain the solution in the transformed domain, in combination with a numerical inversion formula.

\section{FORMULATION OF THE PROBLEM}

Consider a semi-infinite piezoelectric rod occupying the interval $x \geq 0$. At the near end of the rod, a thermal effect is given which raises the temperature of this end to a prescribed temperature with known function and free of stress. Piezoelectric rod direction be parallel with the axial direction. The corresponding boundary conditions may be written as follows [20]:

$$
\left.\theta(x, t)\right|_{x=0}=F(t),\left.\quad \sigma(x, t)\right|_{x=0}=0
$$

In the absence of body force, free charge and inner heat sources, the generalized thermo-piezoelectric governing differential equations as follow:

Equations of motion:

$$
\sigma_{i j, j}=\rho \ddot{u}_{i},
$$

Equation of entropy production (in the absence of inner heat source):

$$
q_{i, i}=-T_{0} \dot{\zeta},
$$

Stress-strain-temperature:

$$
\sigma_{i j}=c_{i j k l} e_{k l}-h_{i j k} D_{k}-\beta_{i j} \theta,
$$

Gauss equation and electric field relation:

$$
\begin{gathered}
D_{i, i}=0, \\
E_{i}=-v_{, i}, \\
E_{i}=h_{i k l} e_{k l}+\tau_{i k} D_{k}-d_{i} T .
\end{gathered}
$$

Equation of entropy:

$$
\zeta=\beta_{i j} e_{i j}+d_{i} D_{i}+c T .
$$

Strain-displacement relations:

$$
e_{i j}=\frac{1}{2}\left(u_{i, j}+u_{j, i}\right)
$$

We will assume the following new form of the heat conduction equation [19]:

$$
q_{i}+A_{i j} \dot{q}_{, j}=-K_{i j} \varphi_{, j},
$$

where $\varphi$ is the conductive temperature and satisfies the relation

$$
\varphi-T=a \varphi_{, i i}
$$

in which $a>0$ is the two-temperature parameter and $k_{i j}$ is the components of thermal conductivity tensor.

Once more, we indicate to the fact that the new modification to the usual theory of generalized thermo piezoelectric is the presence of the conductive temperature into Fourier's law of heat conduction.

In the above equations, a comma followed by a suffix denotes material derivatives and a superposed dot denotes the derivatives with respect to time.

\section{ONE DIMENSION FORMULATION}

For one-dimensional problem we assume displacement component of the form

$$
u_{x}=(x, t), \quad u_{y}=u_{z}=0 .
$$

The following are the linearized basic equations in onedimensional formulation:

$$
\begin{gathered}
(\lambda+2 \mu) \frac{\partial^{2} u}{\partial x^{2}}-\gamma \frac{\partial \theta}{\partial x}=\rho \frac{\partial^{2} u}{\partial t^{2}} \\
\sigma=(\lambda+2 \mu) \frac{\partial u}{\partial x}-\gamma \theta-h D \\
k \frac{\partial^{2} \varphi}{\partial x^{2}}= \\
\left.\frac{\partial}{\partial t}+\tau_{0} \frac{\partial^{2}}{\partial t^{2}}\right)\left[\rho C_{E} \theta+\gamma T_{0} e\right] \\
\varphi-T=a \frac{\partial^{2} \varphi}{\partial x^{2}}, \\
e=\frac{\partial u}{\partial x}, \\
\frac{\partial D}{\partial x}=0 \\
E_{1}=-\frac{\partial v}{\partial x},
\end{gathered}
$$

where, $\gamma=\alpha_{t}(3 \lambda+2 \mu), \alpha_{t}$ is the coefficient of the linear thermal expansion and $x$ is the coordinate taken along the rod, measured from the finite end.

It is convenient now to introduce the following dimensionless variables:

$$
u^{\prime}=c_{0} \eta u, t^{\prime}==c_{0}^{2} \eta t, \quad \sigma^{\prime}=\frac{\sigma}{(\lambda+2 \mu)}
$$




$$
\begin{gathered}
\theta^{\prime}=\frac{T-T_{0}}{T_{0}}, \quad \tau^{\prime}=c_{0}^{2} \eta \tau, \quad \varphi^{\prime}=\frac{\varphi-T_{0}}{T_{0}} \\
D^{\prime}=\frac{h}{\lambda+2 \mu} D, \quad \eta=\frac{\rho C_{E}}{k}, \quad c_{0}^{2}=\frac{\lambda+2 \mu}{\rho}, \\
t_{0}^{\prime}==c_{0}^{2} \eta t_{0}, \quad \Omega^{\prime}==\frac{\Omega}{c_{0}^{2} \eta} .
\end{gathered}
$$

From Gauss's law, since there is no free charge inside the rod, we have $\partial D / \partial x=0$ then it follows that:

$$
D(t)=\text { const. }
$$

Substituting Eq. (16) into Eqs. (12)-(19) and dropping the primes for convenience, we obtain the following set of non-dimensional equations:

$$
\begin{gathered}
\frac{\partial^{2} e}{\partial x^{2}}-\alpha \frac{\partial^{2} \theta}{\partial x^{2}}=\frac{\partial^{2} e}{\partial t^{2}} \\
\sigma=e-\alpha \theta-D \\
\frac{\partial^{2} \varphi}{\partial x^{2}}=\left(\frac{\partial}{\partial t}+\tau_{0} \frac{\partial^{2}}{\partial t^{2}}\right)(\theta+\varepsilon e),
\end{gathered}
$$

and the following relation between the conductive temperature and the thermo dynamical one:

$$
\theta=\varphi-\omega \frac{\partial^{2} \varphi}{\partial x^{2}}
$$

where,

$$
\alpha=\frac{\gamma T_{0}}{(\lambda+2 \mu)}, \quad \varepsilon=\frac{\gamma}{\rho C_{E}}, \quad \omega=a c_{0}^{2} \eta^{2} .
$$

We assume that, the half space $x \geq 0$ is set to be initially at rest and has reference temperature $T_{0}$ such that the initial conditions are assume to be:

$$
\begin{aligned}
& e(x, 0)=\dot{e}(x, 0)=0, \\
& \theta(x, 0)=\dot{\theta}(x, 0)=0, \\
& \varphi(x, 0)=\dot{\varphi}(x, 0)=0 .
\end{aligned}
$$

We consider the half-space $x \geq 0$ at a uniform temperature $T_{0}$ with its boundary $x=0$ subjected to heating with general function $F(t)$ and traction free, so that the boundary conditions take the following forms:

$$
\begin{gathered}
\varphi_{0}(0, t)=F(t), \\
\sigma(0, t)=0,
\end{gathered}
$$

and that

$$
\begin{aligned}
& e(x, t) \rightarrow 0, \\
& \theta(x, t) \rightarrow 0 \text { and } \varphi(x, t) \rightarrow 0 \text { as } x \rightarrow \infty, \quad t>0,
\end{aligned}
$$

Applying Laplace transform defined by:

$$
L\{f(t)\}=\overline{f(s)}=\int_{0}^{\infty} e^{-s t} f(t) d t,
$$

to the both sides of Eqs. (22)-(25), we obtain:

$$
\begin{gathered}
\frac{d^{2} \bar{e}}{d x^{2}}-\alpha \frac{d^{2} \bar{\theta}}{d x^{2}}=s^{2} \bar{e}, \\
\bar{\sigma}=\bar{e}-\alpha \bar{\theta}-\frac{D}{s}, \\
\frac{d^{2} \bar{\varphi}}{d x^{2}}=\left(s+\tau_{0} s^{2}\right) \bar{\theta}+\varepsilon\left(s+\tau_{0} s^{2}\right) \bar{e}, \\
\bar{\theta}=\bar{\varphi}-\omega \frac{d^{2} \bar{\varphi}}{d x^{2}}, \\
\bar{\varphi}(0, s)=\bar{F}(s), \\
\bar{\sigma}(0, s)=0,
\end{gathered}
$$

where s denotes the complex argument related to the Laplace transform.

Eliminating $\bar{\theta}$ between Eqs. (34) and (35), we left with:

$$
\frac{d^{2} \bar{\varphi}}{d x^{2}}=L \bar{\varphi}+L \varepsilon \bar{e}
$$

where

$$
L=L(s)=\frac{s+\tau_{0} s^{2}}{1+\omega\left(s+\tau_{0} s^{2}\right)} .
$$

Substituting from Eq. (38) into Eq. (35), we obtain

$$
\bar{\theta}=(1-\omega L) \bar{\varphi}-\omega L \varepsilon \bar{e}
$$

Eliminating $\bar{\theta}$ between Eq. (32) and Eq. (33) we obtain

$$
\frac{d^{2} \bar{e}}{d x^{2}}=M \bar{\varphi}+N \bar{e}
$$

where

$$
M=M(s)=\frac{\alpha L(1-\omega L)}{1+\omega \alpha \varepsilon L}
$$


and $\quad N=N(s)=\frac{s^{2}+\alpha \varepsilon L(1-\omega L)}{1+\omega \alpha \varepsilon L}$.

\section{STATE SPACE APPROACH}

Equations (38) and (40) can be written matrix differential equations as follows [21]:

$$
\frac{d^{2} \bar{V}(x, s)}{d x^{2}}=A(s) \bar{V}(x, s),
$$

where

$$
\bar{V}(x, s)=\left[\begin{array}{l}
\bar{\varphi} \\
\bar{e}
\end{array}\right]
$$

is the state vector in the transform domain, and, $A(s)$ is a $2 \times 2$ matrix assume the form

$$
A(s)=\left[\begin{array}{cc}
L & \varepsilon L \\
M & N
\end{array}\right] .
$$
form:

The general solution of Eq. (42) can be obtained in the

$$
\bar{V}(x, s)=\exp [-\sqrt{A(s)} x] \bar{V}(0, s)
$$

where for bounded solution with large $x$, we have canceled the exponential part that has a positive power, the matrix exponential $\exp (-\sqrt{A(s)} x)$ is the transfer matrix and

$$
\bar{V}(0, s)=\left[\begin{array}{l}
\bar{\varphi}_{0} \\
\bar{e}_{0}
\end{array}\right] .
$$

where

$$
\bar{\varphi}_{0}=\bar{F}(s)
$$

and from Eqs. (33), (37), (39) and (45), we have

$$
\bar{e}_{0}=\frac{1}{1+\omega L \varepsilon \alpha}\left[\alpha(1-\omega L) \bar{F}(s)+\frac{D}{s}\right],
$$

where

$$
\bar{e}_{0}=\bar{e}(0, s)
$$

The characteristic equation corresponding to the matrix $A$ assumes the form:

$$
\lambda^{2}-(L+N) \lambda+(L N-\varepsilon L M)=0,
$$

The roots of this equation, namely, $\lambda_{1}$ and $\lambda_{2}$, satisfy the following relations

$$
\lambda_{1}+\lambda_{2}=L+N
$$

$$
\lambda_{1} \lambda_{2}=L N-\varepsilon L M
$$

The Taylor series expansion for the matrix exponential $\exp (-\sqrt{A(s)} x)$ is given by

$$
\exp [-A(s) x]=\sum_{n=o}^{\infty} \frac{[-\sqrt{A(s)} x]^{n}}{n !} .
$$

Using Cayley-Hamilton theorem, we can express $A^{2}$ and higher orders of the matrix $A$ in terms of $I, A$, where $I$ is the unit matrix of second order.

Thus, the infinite series in Eq. (49) can be reduced to the following form

$$
\exp [-\sqrt{A(s)} x]=a_{0}(x, s) I+a_{1}(x, s) A(s) .
$$

where $\mathrm{a}_{0}$ and $\mathrm{a}_{1}$ are some coefficients depending on $s$ and $x$.

By Cayley-Hamilton theorem, the characteristic roots $\lambda_{1}$ and $\lambda_{2}$ of the matrix $A$ must satisfy Eq. (50), thus we have

$$
\exp \left(-\sqrt{\lambda_{1}} x\right)=a_{0}+a_{1} \lambda_{1}
$$

and

$$
\exp \left(-\sqrt{\lambda_{2}} x\right)=a_{0}+a_{1} \lambda_{2}
$$

Solving the above linear system of equations, we get

$$
a_{0}=\frac{\lambda_{1} e^{-\sqrt{\lambda_{2}} x}-\lambda_{2} e^{-\sqrt{\lambda_{1}} x}}{\lambda_{1}-\lambda_{2}},
$$

and

$$
a_{1}=\frac{e^{-\sqrt{\lambda_{1}} x}-e^{-\sqrt{\lambda_{2}} x}}{\lambda_{1}-\lambda_{2}} .
$$

Hence, we have

$$
\exp [-\sqrt{A(s)} x]=L_{i j}(x, s), i, j=1,2,
$$

where

$$
\begin{gathered}
L_{11}=\frac{\left(\lambda_{1}-L\right) e^{-\sqrt{\lambda_{2}} x}-\left(\lambda_{2}-L\right) e^{-\sqrt{\lambda_{1}} x}}{\lambda_{1}-\lambda_{2}}, \\
L_{12}=\frac{g L\left(e^{-\sqrt{\lambda_{1}} x}-e^{-\sqrt{\lambda_{2}} x}\right)}{\lambda_{1}-\lambda_{2}}, \\
L_{22}=\frac{\left(\lambda_{2}-N\right) e^{-\sqrt{\lambda_{1}} x}-\left(\lambda_{1}-N\right) e^{-\sqrt{\lambda_{2}} x}}{\lambda_{2}-\lambda_{1}}, \\
L_{21}=\frac{M\left(e^{-\sqrt{\lambda_{1}} x}-e^{-\sqrt{\lambda_{2}} x}\right)}{\lambda_{1}-\lambda_{2}} .
\end{gathered}
$$

We can write the solution in (43) in the following form

$$
\bar{V}(x, s)=L_{i j} \bar{V}(0, s) .
$$


Hence, we obtain

$$
\bar{\varphi}=\varphi_{1} e^{-\sqrt{\lambda_{2}} x}-\varphi_{2} e^{-\sqrt{\lambda_{1}} x},
$$

where

$$
\begin{gathered}
\varphi_{1}=\frac{\left(\lambda_{1} \bar{\varphi}_{0}-L \bar{\varphi}_{0}-g L \bar{e}_{0}\right)}{\lambda_{1}-\lambda_{2}}, \\
\varphi_{2}=\frac{\left(\lambda_{2} \bar{\varphi}_{0}-L \bar{\varphi}_{0}-g L \bar{e}_{0}\right)}{\lambda_{1}-\lambda_{2}},
\end{gathered}
$$

and

$$
\bar{e}=e_{1} e^{-\sqrt{\lambda_{2}} x}-e_{2} e^{-\sqrt{\lambda_{1}} x}
$$

where

$$
\begin{aligned}
& e_{1}=\frac{\left(\lambda_{1} \bar{e}_{0}-M \bar{\varphi}_{0}-N \bar{e}_{0}\right)}{\lambda_{1}-\lambda_{2}}, \\
& e_{2}=\frac{\left(\lambda_{2} \bar{e}_{0}-M \bar{\varphi}_{0}-N \bar{e}_{0}\right)}{\lambda_{1}-\lambda_{2}}
\end{aligned} .
$$

Using Eqs. (57) and (58) into Eq. (39), we obtain

$$
\bar{\theta}=\theta_{1} e^{-\sqrt{\lambda_{2}} x}-\theta_{2} e^{-\sqrt{\lambda_{1}} x},
$$

where

$$
\begin{aligned}
& \theta_{1}=(1-\omega L) \varphi_{1}-\omega L e_{1}, \\
& \theta_{2}=(1-\omega L) \varphi_{2}-\omega L e_{2} .
\end{aligned}
$$

Now, we can get the stress equation by using Eqs. (58) and (59) into Eq. (33), thus we have

$$
\bar{\sigma}=\sigma_{1} e^{-\sqrt{\lambda_{2}} x}-\sigma_{2} e^{-\sqrt{\lambda_{1}} x}-\frac{D}{S},
$$

where

$$
\sigma_{1}=\left(e_{1}-\alpha \theta_{1}\right), \quad \sigma_{2}=\left(e_{2}-\alpha \theta_{2}\right) \text {. }
$$

Which complete the solution on the Laplace transform domain.

\section{Application I (Thermal shock problem)}

We consider the half-space $x \geq 0$ at a uniform temperature $T_{0}$ with its boundary $x=0$ subjected to thermal shock as follows [17]:

$$
F(t)=F_{0} H(t),
$$

where $F_{0}$ is constant represent the strength of the shock on the boundary, and $H(t)$ is the Heavyside unit step function.

After using the Laplace transform, we have

$$
\bar{\varphi}_{0}=\bar{F}(s)=\frac{F_{0}}{s},
$$

and

$$
\bar{e}_{0}=\frac{1}{1+\omega L \varepsilon \alpha}\left[\frac{\alpha(1-\omega L) F_{0}}{s}+\frac{D}{s}\right] .
$$

Thus, we get the complete solution for this application on the Laplace transform domain by using Eqs. (62) and (63) into Eqs. (57)-(60).

\section{Application II (Ramp-type heating)}

We consider the half-space $x \geq 0$ at a uniform temperature $T_{0}$ with its boundary $x=0$ subjected to thermal shock as follows [20]:

$$
F(0, t)=\left\{\begin{array}{ll}
0 & t \leq 0 \\
\frac{F_{0}}{t_{0}} t & 0<t \leq t_{0} \\
F_{0} & t>t_{0}
\end{array},\right.
$$

where $F_{0}$ is constant and $t_{0}$ is the ramp type parameter.

After using the dimensionless and the Laplace transform defined previously, we have

$$
\varphi_{0}=\bar{F}(s)=\frac{F_{0}\left(1-e^{-s t_{0}}\right)}{t_{0} s^{2}},
$$

and

$$
\bar{e}_{0}=\frac{1}{1+\omega L \mathcal{E} \alpha}\left[\frac{\alpha F_{0}(1-\omega L)\left(1-e^{-s t_{0}}\right)}{t_{0} s^{2}}+\frac{D}{s}\right] .
$$

Thus, we get the complete solution for this application on the Laplace transform domain by using Eqs. (65) and (66) into Eqs. (57)-(60).

Application III (Harmonically varying temperature)

We consider the half-space $x \geq 0$ at a uniform temperature $T_{0}$ with its boundary $x=0$ subjected to thermal shock as follows [22]:

$$
F(0, t)=F_{o} e^{i \Omega t}
$$

where $F_{0}$ is constant, $\Omega$ is the angular frequency of thermal vibration and $i=\sqrt{-1}$.

After using the dimensionless and the Laplace transform defined previously, we have

$$
\begin{gathered}
\varphi_{0}=\bar{F}(s)=\frac{F_{0}}{s-i \Omega}, \\
\bar{e}_{0}=\frac{1}{1+\omega L \varepsilon \alpha}\left[\frac{\alpha F_{0}(1-\omega L)}{s-i \Omega}+\frac{D}{s}\right] .
\end{gathered}
$$


Thus, we get the complete solution for this application on the Laplace transform domain by using Eqs. (68) and (69) into Eqs. (57)-(60).

\section{NUMERICAL INVERSION OF THE LAPLACE TRANSFORM}

In order to invert the Laplace transform, we adopt a numerical inversion method based on a Fourier series expansion [23, 24].

By this method the inverse $f(t)$ of the Laplace transform $\bar{f}(s)$ is approximated by

$$
\begin{gathered}
f(t)=\frac{e^{c t}}{t_{1}}\left[\frac{1}{2} \bar{f}(c)+R 1 \sum_{k=1}^{N} \bar{f}\left(c+\frac{i k \pi}{t_{1}}\right) \exp \left(\frac{i k \pi t}{t_{1}}\right)\right], \\
0<t_{1}<2 t,
\end{gathered}
$$

where $N$ is a sufficiently large integer representing the number of terms in the truncated Fourier series, chosen such that

$$
\exp (c t) R 1\left[\bar{f}\left(c+\frac{i N \pi}{t_{1}}\right) \exp \left(\frac{i N \pi t}{t_{1}}\right)\right] \leq \varepsilon_{1},
$$

where $\varepsilon_{1}$ is a prescribed small positive number that corresponds to the degree of accuracy required. The parameter $c$ is a positive free parameter that must be greater than the real part of all the singularities of $\bar{f}(s)$. The optimal choice of $c$ was obtained according to the criteria described in [24].

\section{NUMERICAL RESULTS AND DISCUSSION}

The numerical values of the thermal temperature, the dynamical temperature, stress and strain have been calculated for small time $t=0.25$, for wide range of $x=0.0$ up to $x=2.0$, and for $\tau=0.05$ for the relaxation time. In the calculation process, the following constants are necessary to be known including $F_{0}=1.0, \varepsilon=0.003887$, $\alpha=0.036991, \Omega=10^{-5}, \omega=0.1, D=10^{-7}$ [16]. The numerical results are displayed graphically.

We have three groups of graphs where we have three applications:

The first group (Figs. 1-4) displays the solution of the problem for the thermal shock. It shows the differences between the theory of one temperature generalized thermoelasticity and the theory of two-temperature generalized thermoelasticity. Results of this group are also compared with the correspondence case of Tianhu.

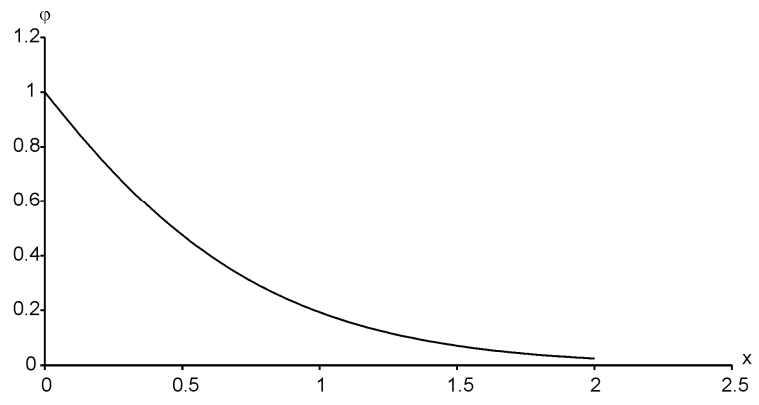

Fig. 1. Heat conduction distribution for thermal shock

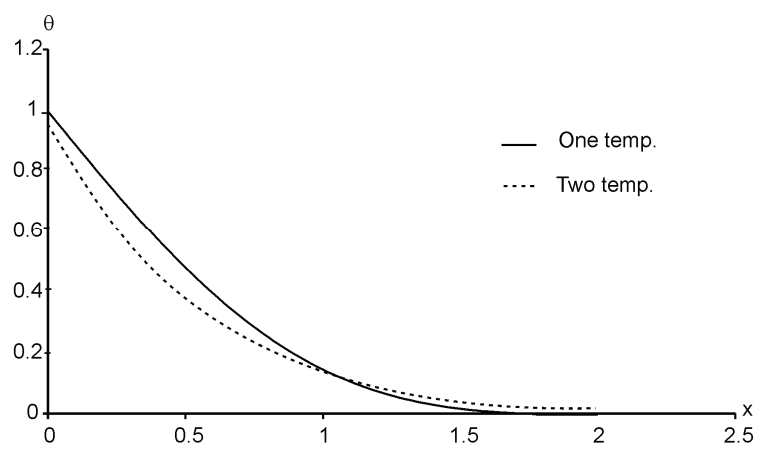

Fig. 2. Dynamical heat conduction distribution for thermal shock

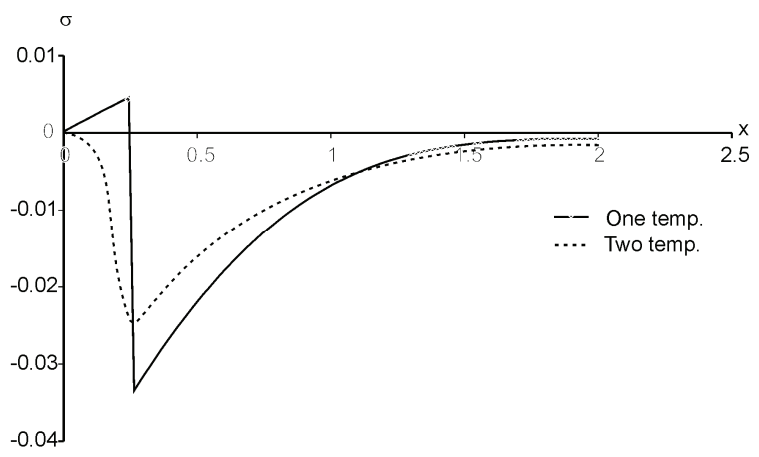

Fig. 3. Stress distribution for thermal shock

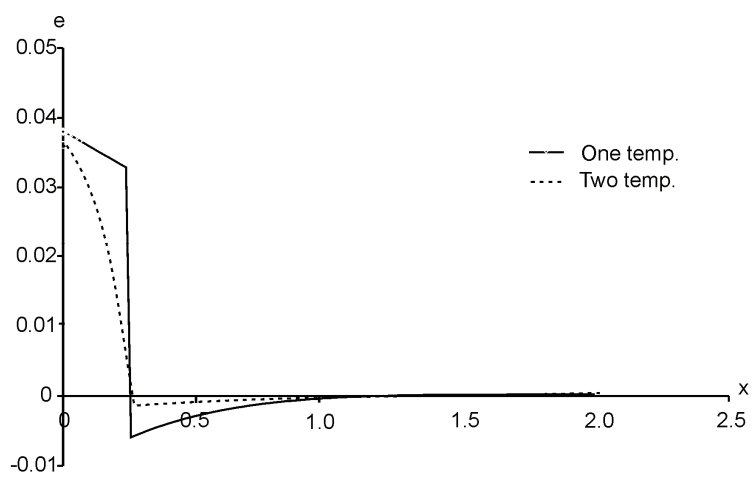

Fig. 4. Strain distribution for thermal shock 
1. Figure 1 displays the conductive temperature and we can deduce that, the wave has a finite speed of propagation. This result shows that the two type temperature model agree with the generalized thermoelasticity.

2. From Fig. 2 we deduce that both type of temperature vanish smoothly far from the nearest end of the rod which is more realistic than the correspondence result of Tianhu which shows that the temperature reduced suddenly to zero.

3. Figure 3 displays a comparison of the stress in the context of the two theories. At $x=0$ the stress reduces to zero which agree with the boundary condition. Comparison with Tianhu work we deduce that the stress distribution is negative in Tianhu work while in the present work we observe that stress in the case of two type temperature is also negative. In the case of one temperature only one jump occur at $x=0.24$ and the magnitude of the stress is 0.0043276 while in the Tianhu work two jumps occur; one of these occurs at $x=0.24$. The two temperature parameter remove the discontinuities appear in the correspondence result of Bassiouny and Tianhu.

4. Figure 4 displays a comparison of the strain in the context of the two theories. We have found that, in the theory of Lord and Shulman the strain distribution has a discontinuous as the stress, while in the theory of Youssef, the strain distribution is continuous and smooth.

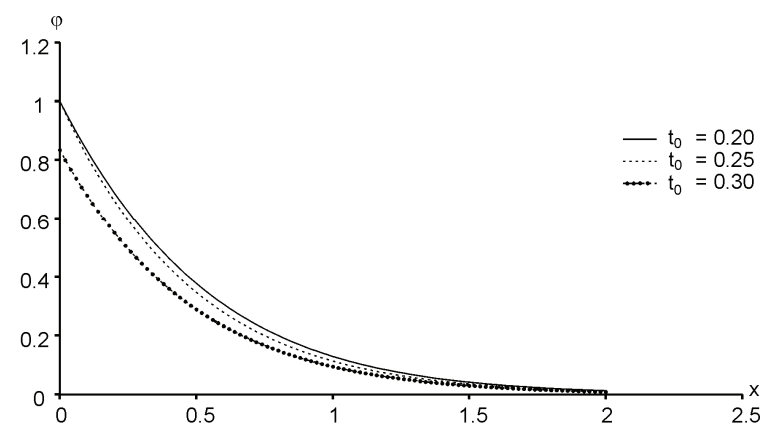

Fig. 5. Heat conduction distribution for ramp-type heating

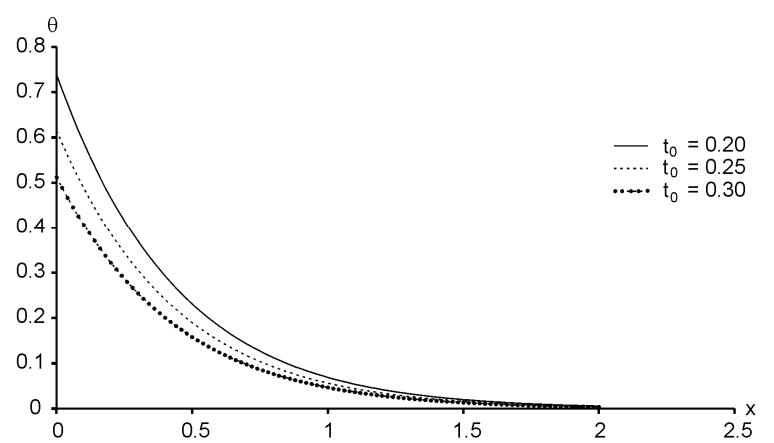

Fig. 6. Dynamical heat distribution for ramp-type heating

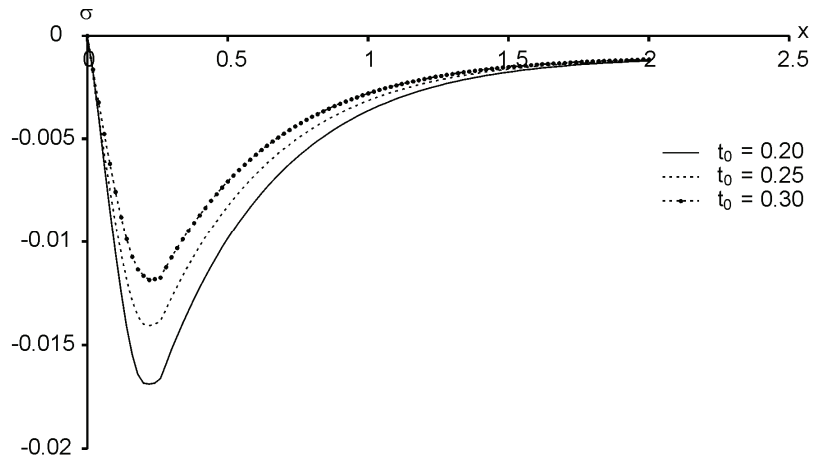

Fig. 7. Stress distribution for ramp-type heating

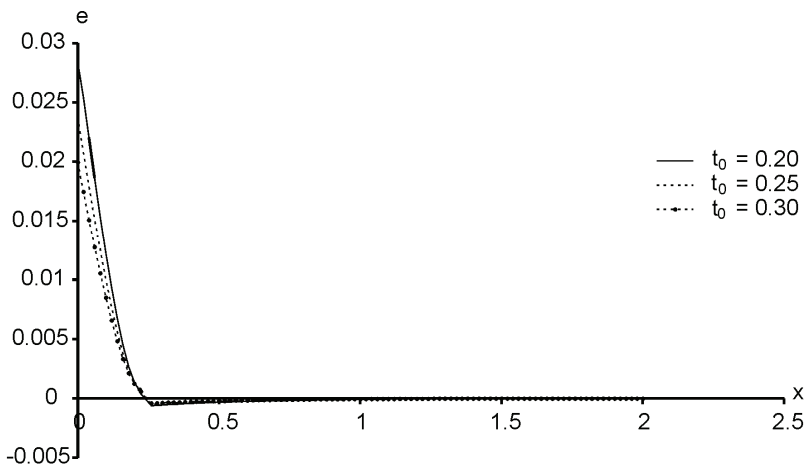

Fig. 8. Strain distribution for ramp-type heating

The second group (Figs. 5-8) displays the solution of the problem for the ramp type heating in the context of Youssef model. This group shows the effect of the ramp type parameter on the results and we found that both type of temperature changes with the same manner due to the changes of the ramp parameter.

The third group (Figs. 9-12) displays the solution of the problem for harmonic heating in the context of Youssef model. This group shows the effect of the relaxation time parameter on the results and we noticed that decreasing the relaxation time increases the heat conductive, the dynami-

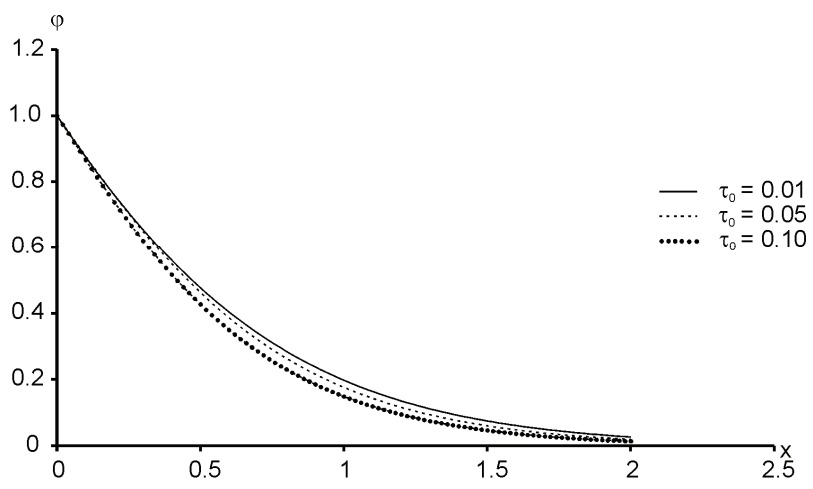

Fig. 9. Heat conduction distribution for harmoically heating 


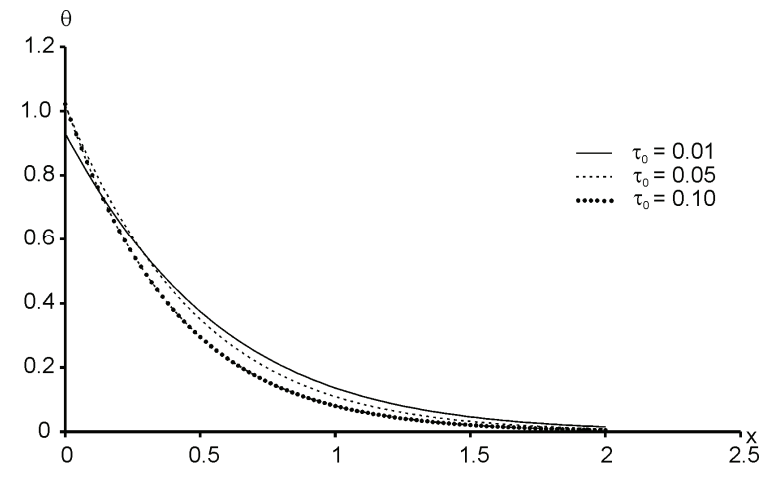

Fig. 10. Dynamical heat conduction distribution for harmoically heating

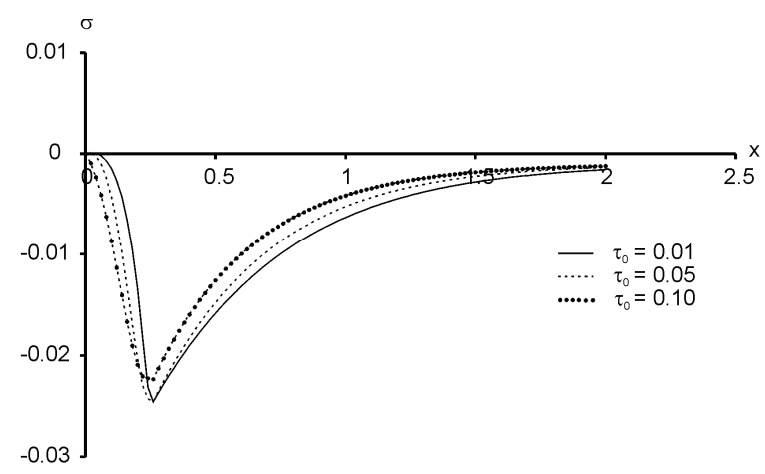

Fig. 11. Stress distribution for harmoically heating

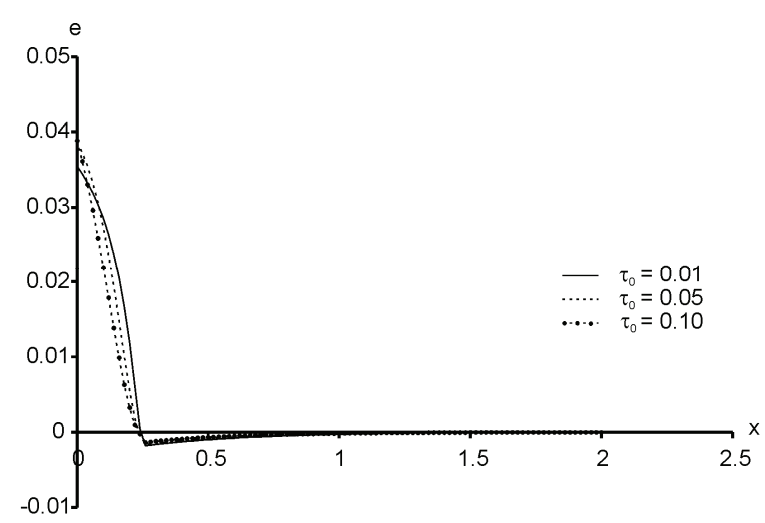

Fig. 12. Strain distribution for harmoically heating

cal heat, the strain and the absolute value of the maximum stress.

\section{CONCLUSION}

Due to the application of two type temperature method to the shock problem of piezoelectric material the discontinuities in the stress and strain function have been re- moved. The way of vanishing the temperature in the present work in comparison with Tianhu work leads us to claim that the present model is more realistic than that of Tianhu. We have found that, the ramp parameter $t_{0}$ as well as the relaxation time $\tau_{0}$ has significant effects on all the fields.

\section{References}

[1] H. W. Lord and Y. Shulman, A generalized dynamical theory of thermoelasticity, J. Mech. Phys. Solids 15, 299-309 (1967).

[2] N. Naotak, R. Hetnarski and Y. Tanigawa, (2003), Thermal Stresses (2nd Edition), Taylor \& Francis, New York.

[3] J. Ignaczak and A Note, On Uniqueness in Thermoelasticity With One Relaxation Time, J. Thermal Stresses 5, 257-263 (1982).

[4] Müller, The coldness, A universal function in thermoelastic solids, Arch. Ration. Mech. An. 41, 319-332 (1971).

[5] Ahmed S. El-Karamany and Magdy A. Ezzat, Thermal shock problem in generalized thermo-viscoelasticty under four theories, Int. J. of Eng. Sci. 42(7) 649-671 (2004).

[6] A. E. Green and K. E. Lindsay, Thermoelasticity, J. Elasticity 2, I-7 (1972).

[7] D. Y. Tzou, Macro- to microscale heat transfer: the lagging behavior, Taylor \& Francis, Washington (1997) DC.

[8] R. D. Mindlin, On the equations of motion of piezoelectric crystals, in: N. I. Muskilishivili, Problems of continuum Mechanics, 70th Birthday Volume, SIAM, Philadelphia, 282-290 (1961).

[9] R. D. Mindlin, Equations of high frequency vibrations of thermo-piezoelectric plate, Int. J. Solids Struct. 10, 625637 (1974).

[10] W. Nowacki, Some general theorems of thermo-piezoelectricity, J. Therm. Stress. 1, 171-182 (1978).

[11] W. Nowacki, Foundations of linear piezoelectricity, in: H. Parkus (Ed), Electromagnetic interactions in Elastic Solids, Springer, Wein, Chapter 1. (1979),

[12] D. S. Chandrasekharaiah, A generalized thermoelastic wave propagation in a semi-infinite piezoelectric rod, Acta Mech. 71, 39-49 (1988).

[13] M. C. Majhi, Discontinuities in generalized thermoelastic wave propagation in a semi-infinite piezoelectric rod, J. Tech. Phys. 36, 269-278 (1995).

[14] J. N. Sharma, M. Kumar, Plane harmonic waves in piezo-thermoelastic materials, Indian Eng. Mater. Sci. 7, 434-442 (2000).

[15] E. Bassiouny and A. F. Gahleb, A one dimensional problem in the generalized theory of thermopiezoelasticity, In: G. A. Maugin (Ed), The mechanical behavior of electromagnetic solid continua, Elsevier science publisher B. V. (North Holland), IUTAM-IUPAP, 79-84 (1984).

[16] H. Tianhu, T. Xiaogeneg and S. Yapeng, State space approach to one-dimensional shock problem for a semiinfinite piezoelectric rod, Int. J. Eng. Sci. 40, 1081-1097 (2002). 
[17] H. Tianhu, T. Xiaogeneg and S. Yapeng, Two-dimensional generalized thermal shock a thick piezoelectric plate of infinite extent, Int. J. Eng. Sci. 40, 2249-2264 (2002).

[18] B. Singh, Wave propagation in an anisotropic generalized thermoelastic solid, Indian J. Pure Appl. Math. 34, 1479-1485 (2003).

[19] H. Youssef, Theory of Two-Temperature Generalized Thermoelasticity, IMA Journal of Applied Mathematics, IMA J. Appl. Math. 71(3), 383-390 (2006).

[20] H. Youssef, Problem of Generalized Thermoelastic Infinite Medium with Cylindrical Cavity Subjected to a Ramp-Type Heating and Loading, Archive Appl. Mech. 75, 553-565 (2006).
[21] L. Y. Bahar and R. B. Hetnarski, State Space Approach to Thermoelasticity, J. Thermal Stresses 1, 135 (1978).

[22] S. Mukhopadhyay, Thermoelastic interactions without energy dissipation in an unbounded body with a spherical cavity subjected to harmonically varying temperature, J. Mech. Res. Comm. 31, 81-89 (2004).

[23] G. Hanig and U. Hirdes, A method for the numerical inversion of Laplace transform, J. Comp. Appl. Math. 10, 113-132 (1984)

[24] H. Chu, C. Chen and C. Weng, Applications of Fourier series Technique to transient heat transfer, Chem. Eng. Commun. 16, 215-227 (1982).

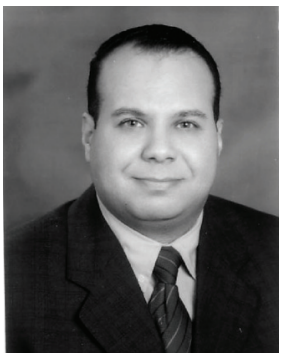

Prof. Hamdy M. Youssef was born on April 22, 1971. In 2003, he received $\mathrm{PhD}$ in Mathematics at Alexandria University, Egypt. His nationality is Egyptian. He is a member of the Egyptian Mathematical Society and IBC Research Council. At present, his affiliation is the Umm Al-Qura University, Saudi Arabia. He works in the field of theory of thermoelasticity. His most important papers are: Theory of TwoTemperature Generalized Thermoelasticity, IMA Journal of Applied Mathematics 71(3), 383-390, 2006 and Theory of generalized porothermoelasticity, International Journal of Rock Mechanics and Mining Sciences 44, 222-227, 2007. His more detailed CV can be found in "Who's Who in Science and Engineering", 2006-2007 and in "2000 Outstanding Intellectuals of the $21^{\text {th }}$ Century", Cambridge University, 2006.

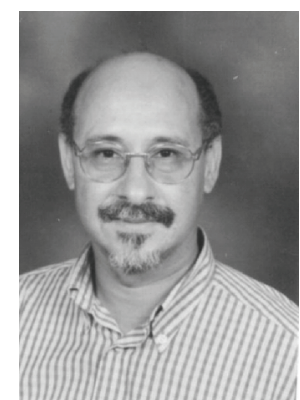

Dr. Essam BassiounY was born on March 30, 1954. He obtained the PhD degree in Mathematics after his studies in "Laboratoire de Mécanique Théorique", Université Pierre-et-Marie Curie Paris, France in 1989. He works in Cairo University, faculty of Science, Fayoum Branch. His affiliation now is The King Saud University, Faculty of Science at Al Kharj, Saudi Arabia. His fields of interest are the Theory of Generalized Thermoelasticity and Damage Mechanics in Ferroelectrics. His most important paper are: E. Bassiouny, Damage Mechanics in Ferroelectric Ceramics - Model and Application, J. Appl. Sci. 5(2): 257-266, 2005; E. Bassiouny, Hamdy M. Youssef. Two Temperature Piezoelectric Generalized Thermoelasticity Finite Rod Subjected to Different Types of Thermal Loading. Journal of Thermal Stresses 31, 1-13, 2008. 